\title{
SCLC im Endstadium: Vorbeugende WBRT?
}

Die prophylaktische Ganzhirnbestrahlung (WBRT) führte nicht zum erhofften Erfolg für Patienten mit kleinzelligem Lungenkarzinom im Stadium IV (SCLC-ED). Sie überlebten nicht länger als nicht bestrahlte Teilnehmer.

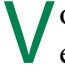
on einer prophylaktischen WBRT erhofft man sich bei Patienten mit SCLC-ED nach palliativer Chemotherapie einen Schutz vor einer zerebralen Metastasierung sowie ein längeres Überleben. Das Vorgehen stützt sich auf die Ergebnisse einer EORTC-Studie und wird in aktuellen internationalen Leitlinien empfohlen. Doch seit einiger Zeit regen sich aufgrund methodischer Mängel der Studie Zweifel.

Japanische Forscher überprüften nun die Effekte der prophylaktischen WBRT erneut in einer Phase-III-Studie an 47 Zentren. Insgesamt 224 Patienten mit SCLC-ED ohne Hirnmetastasen, die auf eine platinbasierte Chemotherapie angesprochen hatten, erhielten entweder im Mittel 37 Tage nach der Chemotherapie eine prophylaktische Hirnradiatio (25 Gy an 10 Tagen à 2,5 Gy) oder wurden ausschließlich beobachtet. Bei allen Probanden war maximal 4 Wochen vor
\end{abstract}

Beginn ein MRT des Gehirns angefertigt worden. Die Patienten wurden im ersten Jahr unabhängig von ihren neurologischen Symptomen im Abstand von 3 Monaten sowie nach 18 und 24 Monaten zu einem MRT aufgefordert.

In der geplanten Zwischenanalyse zu den ersten 163 randomisierten Patienten errechneten die Forscher im Juni 2013 eine Wahrscheinlichkeit von $0,011 \%$, dass die prophylaktische WBRT signifikante Überlebensvorteile gegenüber der reinen Beobachtung bringen würde und brachen die Studie ab.

Die Schlussauswertung bestätigte letztlich die erste Einschätzung: In der Bestrahlungsgruppe lag das Gesamtüberleben im Median bei 11,6 Monaten, in der Beobachtungsgruppe bei 13,7 Monaten. Nach 12 Monaten waren bei $32,9 \%$ der bestrahlten und bei $59 \%$ der beobachteten Teilnehmer Hirnmetastasen diagnostiziert worden.

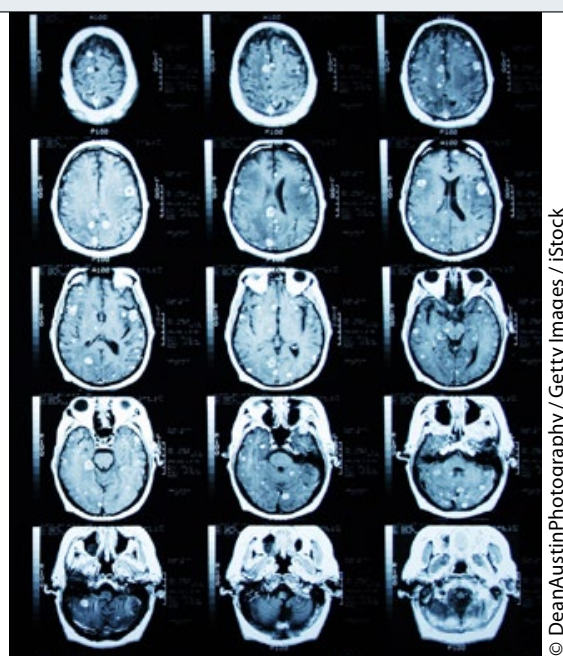

Eine Basis-MRT war ein wichtiges Kriterium des Studiendesigns.

Fazit: Im Gegensatz zur EORTC-Studie konnte in der aktuellen Untersuchung die prophylaktische WBRT das Überleben nicht verlängern. Der wesentliche Unterschied zwischen beiden Studien: In der aktuellen Untersuchung hatte ein MRT zu Studienbeginn Hirnmetastasen ausgeschlossen. In der EORTC-Studie hatten nur $29 \%$ der Teilnehmer ein MRT erhalten.

Christine Starostzik

Takahashi T et al. Prophylactic cranial irradiation versus observation in patients with extensive-disease small-cell lung cancer: a multicentre, randomised, open-label, phase 3 trial. Lancet Oncol. 2017;18(5):663-71.

\section{Bedeutung der Antiangiogenese beim SCLC}

Nach vielversprechenden Ergebnissen in Phase-II-Studien prüften italienische Onkologen in einer Phase-III-Studie die Wirksamkeit und Sicherheit einer Kombination von Bevacizumab mit einer platinhaltigen Standardtherapie beim fortgeschrittenen kleinzelligen Lungenkarzinom (SCLC-ED).

$\mathrm{D}$ ie Patienten erhielten randomisiert maximal 6 Zyklen des Standards Cisplatin plus Etoposid entweder alleine (Arm A) oder kombiniert mit Bevacizumab (Arm B). Trat kein Progress auf, konnte Bevacizumab in Arm B als Erhaltungstherapie bis zum Progress oder für maximal 18 Zyklen weitergegeben werden. Primärer Endpunkt war das Gesamtüberleben (OS). Die Forscher gingen beim Design der Studie optimistisch von einem deutlichen OS-Unterschied in den beiden Armen aus, weshalb nur mit 204 Patienten geplant wurde.
Nach median 34,9 Monaten war das progressionsfreie Überleben (PFS) in Arm B signifikant länger als in Arm A (6,7 vs. 5,7 Monate; $p=0,030$ ). Der Unterschied im OS war nicht signifikant (median 8,9 vs. 9,8 Monate), die 1-Jahres-Überlebensraten betrugen $25 \%$ bzw. $37 \%$ (Hazard Ratio [HR] 0,78; $\mathrm{p}=0,113$ ). Nur in der Subgruppe der Patienten mit Bevacizumab-Erhaltungstherapie gab es einen OS-Vorteil (HR 0,60; $p=0,011$ ).

Die hämatologische Toxizität beider Regimes unterschied sich nicht signifikant. An nicht hämatologischen Toxizi- täten trat im Bevacizumab-Arm nur eine Hypertonie häufiger auf - vom Grad 3/4 bei 6,3\% der Patienten im Arm $B$ und bei $1,0 \%$ der Patienten im Arm A $(\mathrm{p}=0,057)$.

Fazit: Ein verbessertes PFS durch die $\mathrm{Zu}$ gabe von Bevacizumab zu einer platinhaltigen Standardtherapie des SCLC -ED übersetzte sich nicht in ein besseres OS - der primäre Endpunkt der Studie wurde nicht erreicht. Dennoch sehen die Forscher eine Chance für die Antiangiogenese als Therapieprinzip, möglicherweise als Erhaltungstherapie oder mittels neuer antiangiogen wirksamer Substanzen.

Friederike Klein

Tiseo M et al. Italian, Multicenter, Phase III, Randomized Study of Cisplatin Plus Etoposide With or Without Bevacizumab as First-Line Treatment in Extensive-Disease Small-Cell Lung Cancer: The GOIRC-AIFA FARM6PMFJM Trial. J Clin Oncol. 2017;35(12):1281-7. 